\title{
Anti-Fibrotic Effects of Astragaloside IV in Systemic Sclerosis
}

\author{
Qing Qia Yueping Mao Juanjuan Yib Donghai $\mathrm{Li}^{\mathrm{a}} \quad \mathrm{Ke} \mathrm{Zhu}^{\mathrm{a}} \quad \mathrm{Xushan}^{\mathrm{b}}$ Cha \\ aDepartment of Dermatology, The First Affiliated Hospital, Guangzhou University of Chinese Medicine, \\ Guangzhou, 'Department of Dermatology, Sun Yat-Sen Memorial Hospital, Sun Yat-Sen University, \\ Guangzhou, China
}

\section{Key Words}

Systemic sclerosis (SSc) - Fibrosis - Transforming growth factor $\beta$ (TGF- $\beta$ ) Collagen I • Fibronectin

\begin{abstract}
Objective: To evaluate the anti-fibrotic effects of Astragaloside IV in systemic sclerosis. Methods: Treated or untreated systemic sclerosis (SSc) and normal fibroblast isolated from corresponding pairs were utilized to detect expression of collagen and fibronectin by western blot, quantitative real-time RT-PCR (RT-qPCR), immunofluorescence staining and histopathological examination. SSc mouse model induced by bleomycin was used to evaluate the effects of the drug in vivo. Results: Compared to normal fibroblast (NF), the expression of collagen and fibronectin in SSc (SSCF) dramatically increased, and this could be reduced by Astragaloside IV (AST) in a dose- or time-dependent manner at both protein and mRNA levels. Administration of Astragaloside IV consistently decreased collagen formation and partially restored the structure, as well as suppressing collagen and fibronectin expression in the skin lesions of SSc-model mice. Mechanistically, Astragaloside IV-induced fibrosis reduction may be due to deregulation of Smad 3/Fli-1, the major mediators of the fibrotic response and key molecules for TGF- $\beta$ signaling. Astragaloside IV also decreased the level of p-SMAD3 and completely blocked its relocation into the nuclei. Conclusion: Astragaloside IV attenuates fibrosis by inhibiting the TGF- $\beta$-Smads3 axis in systemic sclerosis.
\end{abstract}

Copyright $\odot 2014$ S. Karger AG, Basel

\section{Introduction}

Systemic sclerosis (SSc) is a complex, heterogeneous, autoimmune connective tissue disease characterized by excessive fibrosis of the skin lesions and internal organs due to fibroblast proliferation and excessive production of the extracellular matrix (ECM) [1]. The disease has two major sub-groups, classified as limited cutaneous scleroderma (ISSc), and 
diffuse cutaneous scleroderma (dSSc) [2]. There is currently no effective cure for this disease. However, since fibrosis is a major cause of morbidity and mortality in SSc [3], studies suggest that the therapeutic targeting of fibrosis may be one of the potential options in treating SSc [4]. The transforming growth factor $\beta$ (TGF- $\beta$ ), associated with the Smad pathway, plays a fundamental role in pathological fibrogenesis in SSc. Smads are intracellular proteins that transduce extracellular signals from TGF- $\beta$-like ligands, which are themselves grouped into three subfamilies: five receptor-activated Smads (R-Smads), one common mediator Smad (Co-Smad), and two inhibitory Smads. Among R-Smads, Smads 2 and 3 signal for TGF- $\beta$ and activin, while Smads 1, 5 and 8 transduce signals from BMP ligands [5]. Studies show that elevated expression of TGF- $\beta$ and its aberrant regulated genes as well as heightened activity in its signaling pathway was present in SSc-related progressive lung fibrosis [6] and dermal fibrosis [7]. Lakos et al. found that compared to fibroblasts derived from wild mice, Smad3null fibroblasts showed reduced in vitro proliferative and profibrotic responses elicited by TGF- $\beta$ [8], suggesting that drugs interfering with TGF- $\beta$-Smads may be promising in SSc treatment.

Astragaloside IV, a new glycoside of cycloartane-type triterpene, is one of the chief effective components isolated from Astragalus membranaceus, a Chinese medical herb that has long been widely used for the management of various diseases [9]. It has been proved to function as an anti-inflammatory through inhibiting NFKB-mediated inflammatory genes expression [10], is useful in treating myocardial injury [11], and also has immunoregulatory properties, all without evident toxicity or side effects $[12,13]$. Interestingly, recent studies have shown that Astragaloside IV also has potent anti-fibrotic effects. Liu et al. found Astragaloside IV displayed antifibrotic effects in rats induced though porcine-serum, the mechanism of which might be associated with its inhibitory effects on collagen synthesis and proliferation in hepatic stellate cells [14]. Astragaloside IV consistently suppressed collagen production in activated hepatic stellate cells via the oxidative stress-mediated p38 MAPK pathway [15]. Further, Astragaloside IV attenuates myocardial fibrosis by inhibiting TGF- $\beta$ signaling [11] and exerts healing and anti-scar effects useful in wound treatment $[16,17]$. However, information about the efficacy of Astragaloside IV in the treatment of systemic sclerosis (SSc) is limited.

Thus, in this study we aimed to evaluate anti-fibrotic effects of Astragaloside IV in systemic sclerosis and revealed the underlying mechanism.

\section{Material and Methods}

\section{Ethics statement}

All our experiments involving human participants were approved by the Medical Research Ethics Committee of the First Affiliated Hospital of Guangzhou University of Chinese Medicine, and conducted according to the National Institutes of Health (NIH) Guide and Declaration of Helsinki (2004). All participants involved in the study signed informed consent forms and all animal experiments were conducted according to relevant national and international guidelines. This project was approved by the Medical Research Animal Ethics Committee of Guangzhou University of Chinese Medicine.

\section{Patient samples and cell culture}

After informed consent was provided and in compliance with the Institutional Review Board of Human Studies, $5 \mathrm{~mm}$ skin biopsy specimens were obtained from affected areas (dorsal forearm) of 8 patients (3 females and 5 males) with diffuse cutaneous SSc (Table 1). All patients fulfilled the American College of Rheumatology (formerly, the American Rheumatism Association) criteria for SSc [18]. Dermal fibroblasts were obtained from the biopsy samples by enzymatically dissociating tissue specimens in $0.25 \%$ type I collagenase (Sigma, St. Louis, MO, USA) and 0.05\% DNase (Sigma) in Dulbecco's modified Eagle's medium (DMEM, Gibco, Carlsbad, CA, USA) with $20 \%$ fetal bovine serum (HyClone, Logan, UT, USA). Cells were grown in DMEM supplemented with $10 \%$ fetal calf serum and $50 \mathrm{~g} / \mathrm{ml}$ gentamicin (Sigma) at $37^{\circ} \mathrm{C}$ in a $5 \% \mathrm{CO}_{2}$ atmosphere. Normal control dermal fibroblasts were derived from foreskin biopsy specimens of 10 healthy 


\begin{tabular}{|c|c|c|}
\hline Cellı & Cell Physiol Biochem 2014;34:2105-2116 & \\
\hline and Biochemistry & $\begin{array}{l}\text { DOI: } 10.1159 / 000366405 \\
\text { Published online: November 28, } 2014\end{array}$ & $\begin{array}{l}\text { (c) } 2014 \text { S. Karger AG, Basel } \\
\text { www.karger.com/cpb }\end{array}$ \\
\hline
\end{tabular}

Table 1. Patient characteristics

\begin{tabular}{ccccccc}
\hline \multirow{5}{*}{ SSc patient } & & Gender & Age & Ethnic Origin & Diagnosis & Skin Biopsy Position \\
& S1 & F & 32 & Han & Diffuse SSc & Trunk \\
& S2 & M & 27 & Han & Diffuse SSc & Leg \\
S3 & M & 25 & Han & Limited SSc & Leg \\
& S4 & F & 30 & Han & Limited SSc & Arm \\
S5 & F & 28 & Han & Limited SSc & Arm \\
S6 & M & 41 & Han & Diffuse SSc & Arm \\
S7 & M & 22 & Han & Limited SSc & Leg \\
S8 & M & 35 & Han & Diffuse SSc & Arm \\
Health control & & & & & \\
H1 & M & 30 & Han & & \\
H2 & M & 26 & Han & & \\
H3 & M & 18 & Han & & \\
H4 & M & 24 & Han & & \\
H5 & M & 34 & Han & & \\
H6 & M & 36 & Han & & \\
H7 & M & 23 & Han & & \\
H8 & M & 21 & Han & & \\
H9 & M & 23 & Han & & \\
H10 & M & 22 & Han & & \\
\hline
\end{tabular}

male donors with circumcision surgery who were matched with the SSc patients for age, sex, and race (Table 1). SSc and normal fibroblast pairs were utilized at passages $4-6$ for all experiments.

\section{Bleomycin-induced skin fibrosis mice model and Astragaloside IV treatment}

6-8-week-old female specific pathogen-free (SPF) C57/BL6 mice were supplied by the Laboratory Animal Center of Sun Yat-sen University. The animals were allowed free access to water and standard mouse chow and housed at $25^{\circ} \mathrm{C}$ with $40 \%$ humidity and with a $12 \mathrm{~h} / 12 \mathrm{~h}$ light/dark cycle. The mice were randomly divided into three groups: the control group, the model group, and the Astragaloside IV (AS-IV, Sigma) ( 2.0 and $4.0 \mathrm{mg} / \mathrm{kg}$ ) treatment groups ( $n=6$ mice per group). To establish the skin fibrosis model, bleomycin (Sigma) was diluted to $0.1 \mathrm{U} / \mathrm{ml}$ with PBS, and filter sterilized. $100 \mu \mathrm{l}$ of bleomycin (for model group and AS-IV treatment group) or PBS (for normal group) was injected subcutaneously into a single location on the shaved back of mice once daily for 4 weeks, in accordance with a previous study [19]. To evaluate the effect of Astragaloside IV in vivo, AS-IV solution was prepared by ultrasonication in $0.5 \%$ sodium carboxymethylcellulose (CMC-Na) solution. AS-IV was administered to AS-IV treatment group by gavage once daily for 4 weeks. The same volume of vehicle was given to the normal group and model group as controls. Mice were sacrificed - humanely using $\mathrm{CO}_{2}$, and the lesional skin was collected for further analysis.

\section{Protein extraction and immunoblotting}

The procedures were performed as previously described [20]. In brief, skin samples from the injection site of the animals were homogenized and lysed in lysis buffer containing $7 \mathrm{~mol} / \mathrm{L}$ urea, $2 \mathrm{~mol} / \mathrm{L}$ thiourea, $2 \%$ CHAPS, $0.1 \mathrm{~mol} / \mathrm{L}$ DTT, $0.1 \% \mathrm{NP} 40$, and $40 \mathrm{mmol} / \mathrm{L}$ Tris-HCl using a Polytron homogenizer, followed by centrifugation $(100,000 \mathrm{~g})$ for $30 \mathrm{~min}$ at $4^{\circ} \mathrm{C}$. For the cultured cells, the lysates were harvested by centrifugation $(12,000 \mathrm{~g})$ at $4^{\circ} \mathrm{C}$ for $15 \mathrm{~min}$. The supernatants were separated, and protein concentration was assessed using the Bio-Rad protein assay (Bio-Rad Laboratories, Hercules, CA, United States). After incubation at $100^{\circ} \mathrm{C}$ for $10 \mathrm{~min}$, equal amounts of denatured protein were resolved on a SDS-PAGE and were electrophoretically transferred onto PVDF membranes. The blots were probed with primary antibodies, including anti-collagen, anti-Fli-1 and anti-Fibronectin (Santa cruz, Biotechnology, CA, United States); antiSMAD3 and anti-p-SMAD3 (Cell Signaling Technology, Beverly, MA, United States). After washing, appropriate horseradish peroxidase-conjugated secondary antibodies (Jackson ImmunoResearch, West Grove, PA, USA) were used for detection with the ECL and ECL-plus systems (Forevergen Biosciences, Guangzhou, China). On completion, the membranes were stripped and probed with a $\beta$-actin antibody (Cell Signaling Technology), which served as a loading control. The intensity was quantitated by densitometry.

\section{Quantitative real-time RT-PCR (RT-qPCR)}

The procedure was slightly modified in accordance with previous studies [21, 22]. Total RNA was

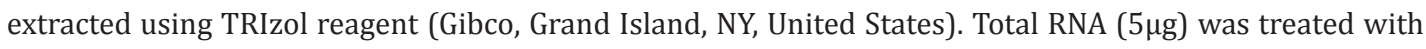


2 units DNase I (bovine pancreas; Sigma) for $15 \mathrm{~min}$ at room temperature in an $18 \mu$ l volume containing $1 \times$ PCR buffer and $2 \mathrm{mM} \mathrm{MgCl}_{2}$, followed by inactivation of DNase I with $2 \mu \mathrm{l}$ of $25 \mathrm{mM} \mathrm{EDTA}$ at $65^{\circ} \mathrm{C}$ for $15 \mathrm{~min}$. Random hexamer primers ( $2 \mu \mathrm{l}$; Promega, Madison, WI, United States) were added and annealed to the RNA according to the manufacturer's protocol. Complementary DNA was synthesized in a $50 \mu$ l reaction volume containing $5 \mu \mathrm{g}$ of total RNA and 50 units Moloney murine leukemia virus RT (Promega) by incubating the tubes at $42^{\circ} \mathrm{C}$ for $45 \mathrm{~min}$. PCR amplification was carried out in $25 \mu \mathrm{l}$ of reaction mixture with $2.0 \mu \mathrm{l}$ of cDNA, $0.5 \mathrm{mM}$ of each primer, and TaKaRa Taq DNA Polymerase (TaKaRa Shuzo, Shiga, Japan) according to the manufacturer's instructions. The primer sets were as follows: GAPDH Forward AATCCCATCACCATCTTCCAG and Reverse AAATGAGCCCCAGCCTTC; Collagen I $\alpha 2$ (COL I $\alpha 2$ ) Forward AGAGTGGAGCAGTGGTTACTA and Reverse GATACAGGTTTCGCCAGTAGAG; Fibronectin Forward GAACTATGATGCCGACCAGAA and Reverse CTGATCTCCAATGCGGTACAT. The Ct (threshold cycle) value of each sample was calculated, and the relative expression was normalized to the expression of GAPDH.

\section{Immunofluorescence staining}

Procedures were in accordance with previous protocols [23]. Cells cultured in chamber slides (Thermo Fisher Scientific, Waltham, MA, United States) were washed, fixed, and permeabilized. The cells were then incubated with rabbit anti-human collagen I antibody (1:50 dilution), or rabbit anti-human fibronectin antibody (1:25 dilution; Novus Biologicals, Littleton, CO, United States). Subsequently, the cells were incubated with fluorescent secondary antibody Alexa Fluor $₫ 488$ Donkey Anti-Rabbit IgG (Invitrogen, Carlsbad, CA, United States), then counterstained with Hoechst 33258 (Invitrogen) for nuclei staining. They were inspected and photographed using fluorescence microscopy (Olympus BX51W1, Tokyo, Japan). Fluorescent positive rates were counted in five randomly selected areas and analyzed independently by three reviewers.

\section{Histopathological examination}

Lesion skin tissues were fixed overnight with $10 \%$ formalin, dehydrated, and embedded in paraffin using standard techniques [24]. Consecutive $5 \mu \mathrm{m}$ serial sections were cut and stained with hematoxylin and eosin (H\&E). To determine the collagen content and organization of the lesional skin, deparaffinized sections were processed for Masson's trichrome stain according to the manufacturer's instructions.

Statistical Analysis

SPSS version 13.0 (SPSS Inc., Chicago, IL) was used for data analysis. Continuous variables with normal distribution are presented as the mean \pm standard deviation (Mean \pm SD). Significance was defined as $\mathrm{P}<0.05$.

\section{Results}

Inhibition of collagen and fibronectin expression by Astragaloside IV in SSc fibroblasts

Fibroblasts from normal (NF) and SSc (SScF) samples were treated with Astragaloside IV (AST) in a variety of doses and durations, followed by western blot and RT-qPCR assays. Compared to normal fibroblasts, the expression of collagen and fibronectin in SSc dramatically increased. When treated with Astragaloside IV, the expression level was decreased in a dose(Fig $1 \mathrm{~A}$ ) and time-dependent manner (Fig. $1 \mathrm{~B}$ ). The expression of collagen and fibronectin in SSc fibroblast shown decreases after treatment with Astragaloside IV in the dose of $30 \mu \mathrm{M}$ for $72 \mathrm{~h}$ (Fig 2). The mRNA level of collagen and fibronectin were consistently significantly higher in SSc fibroblasts than in normal fibroblasts. Treatment with Astragaloside IV suppressed Collagen I $\alpha 2$ (COL I $\alpha 2$ ) and fibronectin mRNA expression in a similar fashion (Fig. 1 C, 1 D). These data suggest that Astragaloside IV has an inhibitory effect on collagen and fibronectin expression in SSc fibroblasts.

\section{Astragaloside IV decrease fibronectin in SSc fibroblasts}

Fibronectin is assembled into the extracellular matrix, and is involved in cell adhesion, growth, migration, and differentiation. Staining fibronectin helps to define fibroblast function. Thus, we utilized immunofluorescence staining to observe the formation of fibronectin. As 


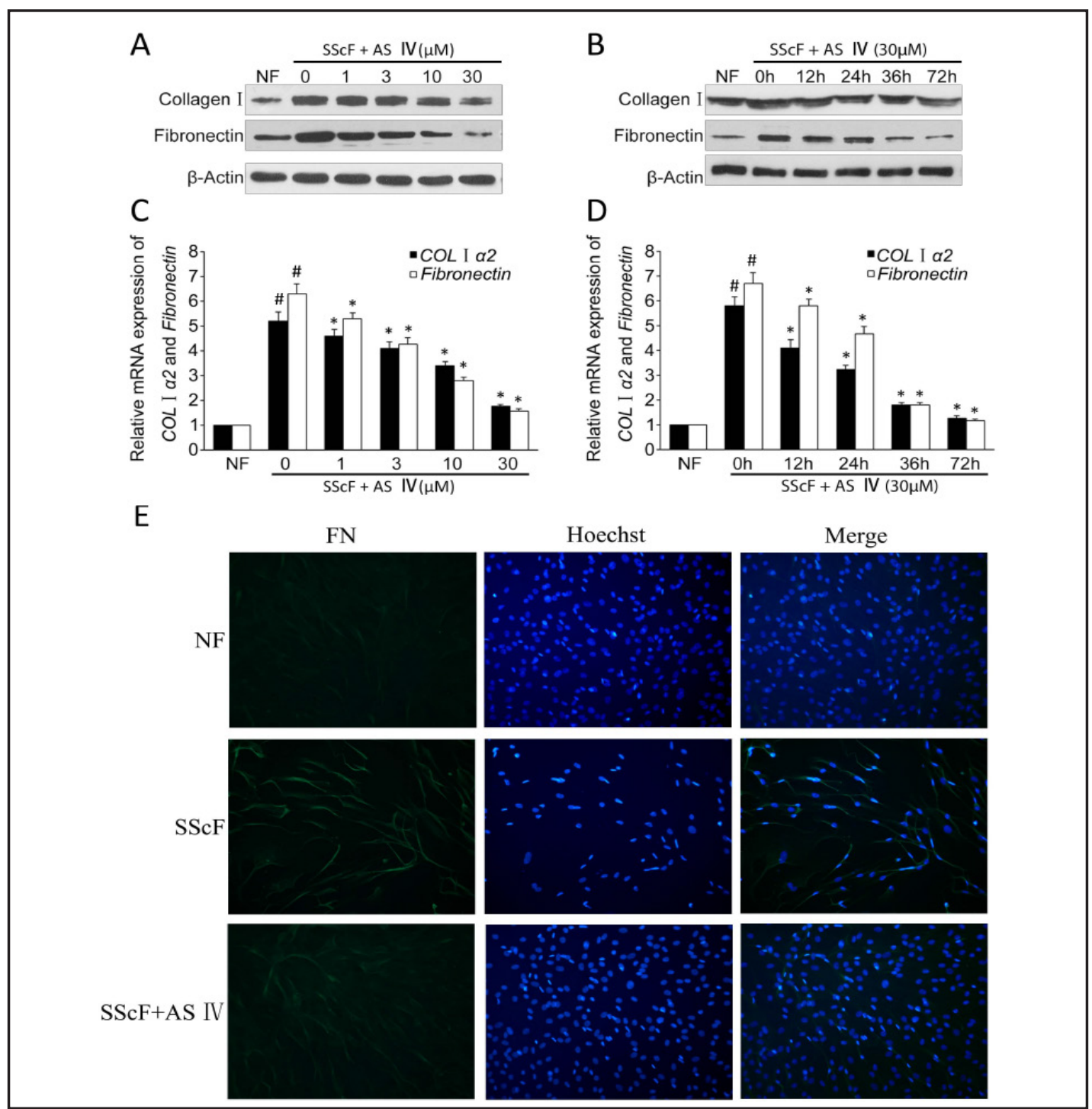

Fig. 1. Inhibition of collagen and fibronectin expression by astragaloside IV in SSc fibroblasts. SSc and normal fibroblasts isolated from corresponding pairs were utilized at passages 4-6 for all experiments. Normal fibroblast (NF) and SSc fibroblast (SScF) were treated with Astragaloside IV (AS IV) in a series of doses (0, $1,3,10,30 \mu \mathrm{M}$ for $72 \mathrm{~h})$ and durations $(0,12,24,36,72 \mathrm{~h}$ at a dose of $30 \mu \mathrm{M})$, followed by western blot and RT-qPCR assays. (A) Compared to normal fibroblast (NF), protein expression of collagen and fibronectin in SSc (SScF) dramatically increased, and was reduced by Astragaloside IV (AS IV) in a dose-dependent manner. (B) Protein expression of collagen and fibronectin in SSc (SScF) was reduced by Astragaloside IV (AST) in a time-dependent manner. (C, D) The mRNA expression of collagen I $\alpha 2$ (COL I $\alpha 2$ ) and fibronectin in SSc (SScF) was consistently reduced by Astragaloside IV (AS IV) in a dose or time-dependent manner. Results are expressed as means \pm SD. Data shown represent at least three patients with three triplicates and are analyzed with ANOVA. ${ }^{*} p<0.05$ vs SScF + AS IV $(0 \mu \mathrm{M})$, \#p $<0.05$ vs NF. (E) Astragaloside IV decreased fibronectin in SSc fibroblasts. Cells cultured in chamber slides were washed, fixed, and permeabilized, followed by immunofluorescence staining. Normal fibroblast (NF) expressed low level of fibronectin. SSc fibroblasts (SScF) expressed high levels of fibronectin with filiform staining and clear borders, which was reduced and decomposed into diffusion with the treatment of Astragaloside IV (SScF + AS IV). Nuclei were stained by Hoechst 33258. NF, normal fibroblast; SScF, SSc fibroblast; AS IV, Astragaloside IV.

shown in Fig. 1E, low levels of fibronectin are expressed in normal fibroblasts, but these increase markedly in SSc fibroblasts. Filiform staining of fibronectin with clear borders 


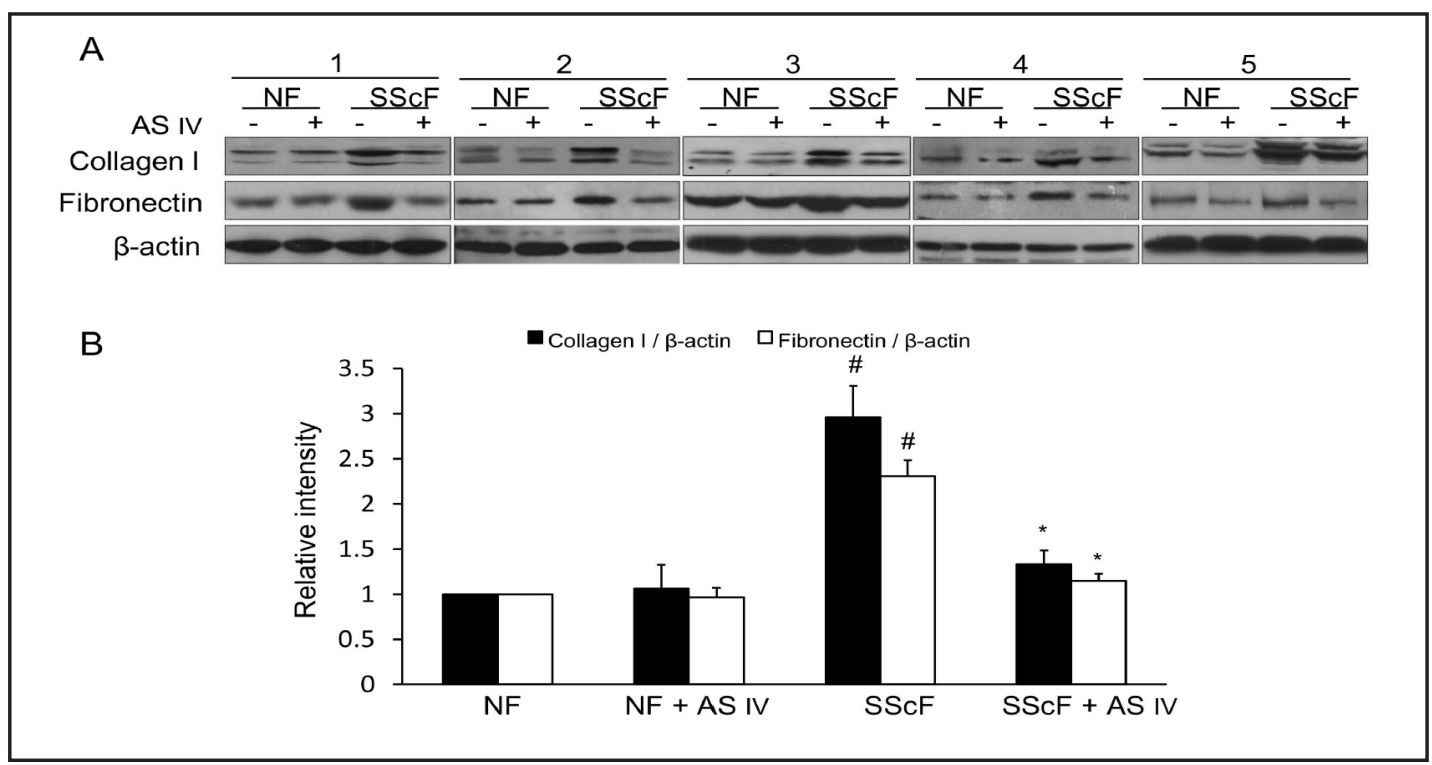

Fig. 2. Collagen and fibronectin expression in fibroblasts from normal and SSc samples treated with Astragaloside IV. (A) Western blot analysis of the expression of collagen and fibronectin in normal fibroblast $(\mathrm{NF})$ and SSc fibroblast (SScF) treated with (+) or without (-) Astragaloside IV ( $\mathrm{n}=5$ for each group). (B) Analyses of band intensity on films are presented as the relative ratio of collagen and fibronectin to actin. Data shown were normalized to NF group. NF + AS IV, normal fibroblast treated with Astragaloside IV in the dose of $30 \mu \mathrm{M}$ for $72 \mathrm{~h}$; SScF, SSc fibroblast treated with Astragaloside IV in the dose of 30 $\mu \mathrm{M}$ for $72 \mathrm{~h}$. Data are expressed as means $\pm \mathrm{SD}$ and analyzed with $\mathrm{ANOVA}^{*} p<0.05$ vs SScF, $\# p<0.05$ vs NF.

was observed in SSc fibroblasts, which decreased and decomposed into diffusion with the treatment of Astragaloside IV.

\section{Inhibition of fibrosis by Astragaloside IV in SSc mouse model}

To test the anti-fibrosis effects of Astragaloside IV in vivo, SSc model mice induced by bleomycin were treated with Astragaloside IV. Lesion skin tissues were subjected to Masson's trichrome as well as H\&E staining. Masson's trichrome is a three-color staining protocol used in histology which produces red keratin and muscle fibers, blue or green collagen and bone, light red or pink cytoplasm, and dark brown to black cell nuclei. As shown in Fig. 3A, normal skin staining exhibited clear layers of epidermis and dermis, with abundant adipocytes in the hypodermis. In contrast, SSc lesion skin displayed massive green collagen staining and abnormal histology structure. Administration of Astragaloside IV decreased the collagen formation as well as partially restored the structure, in a dose-dependent fashion.

Delivery of Astragaloside IV decreased expression of collagen and fibronectin in vivo

To further confirm that the expression of collagen and fibronectin in vivo was reduced, lesion tissues were subjected to western blot and RT-qPCR assays. Consistent with the aforementioned staining results, Collagen I and Fibronectin expression was significantly higher in SSc model samples compared with control, which was in turn attenuated by treatment with Astragaloside IV in dose-dependent manner at both protein (Fig.3B) and mRNA (Fig. 3C) levels.

Astragaloside IV-induced fibrosis reduction is due to deregulation of Smad3/Fli-1

To elucidate the underlying mechanism of Astragaloside IV-induced fibrosis reduction, Smad3/Fli-1 expression, which functions as a mediator of the fibrotic response and comprises key molecules for TGF- $\beta$ signaling, was evaluated. As expected, phosphorylation of Smad3 was suppressed by Astragaloside IV in dose- and time-dependent manner in SSc 


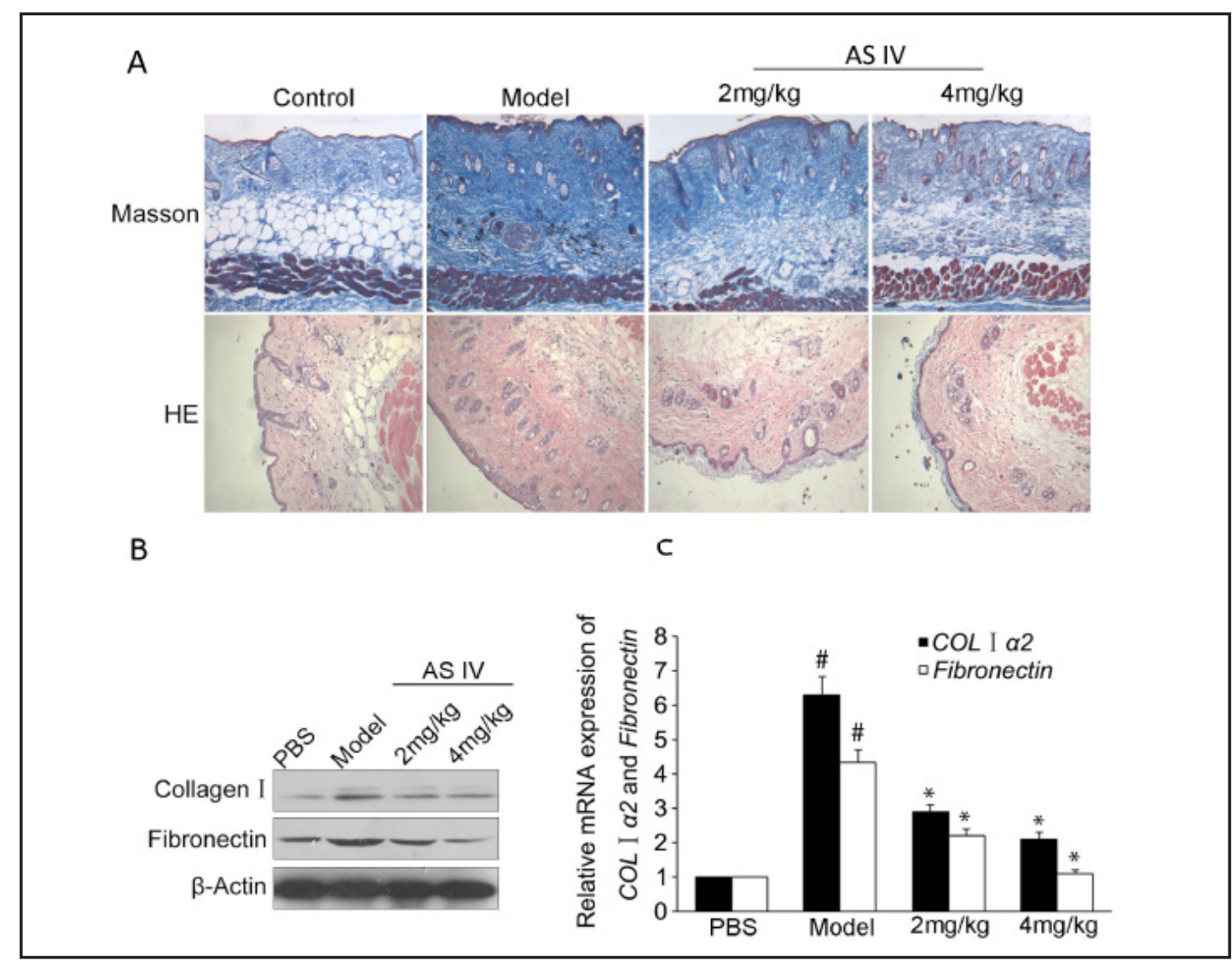

Fig. 3. Inhibition of fibrosis by Astragaloside IV in SSc mouse model. (A) SSc model mice induced by bleomycin were treated with Astragaloside IV. Paraffin-embedded lesion skin tissues were subjected to Masson's trichrome as well as H\&E staining. Normal skin (PBS) staining exhibited clear layers of epidermis and dermis, with abundant adipocytes in hypodermis, while SSc lesion skin (model) displayed massive green collagen staining and abnormal histology structure. Treatment with $2 \mathrm{mg} / \mathrm{kg}$ or $4 \mathrm{mg} / \mathrm{kg}$ Astragaloside IV for 4 weeks dramatically decreased the collagen formation as well as partially restored the structure. (B) Collagen I and Fibronectin protein expression was significantly higher in SSc model compared with control, and was attenuated by treatment of Astragaloside IV at a dose of $2 \mathrm{mg} / \mathrm{kg}$ or $4 \mathrm{mg} / \mathrm{kg}$. (C) mRNA level of collagen I $\alpha 2$ (COL I $\alpha 2$ ) and fibronectin were analyzed by RT-qPCR and displayed the same trend as protein. RT-qPCR results are expressed as means \pm SD of three independent experiments analyzed with ANOVA. ${ }^{*} p<0.05$ vs Model, $\# p<0.05$ vs PBS. PBS, Normal skin, Model, SSc lesion skin, $2 \mathrm{mg} / \mathrm{kg}$, SSc lesion skin treated with $2 \mathrm{mg} / \mathrm{kg}$ Astragaloside IV, $4 \mathrm{mg} / \mathrm{kg}$, SSc lesion skin treated with $4 \mathrm{mg} / \mathrm{kg}$ Astragaloside IV.

fibroblast, while the expression of Fli-1 displayed the opposite trend. No significant change was observed in total Smad3 expression (Fig. 4A, B). Similar results were obtained for their mRNA expression by RT-qPCR (Fig. 4C, D). These data suggest that Astragaloside IV-induced fibrosis reduction may be due to deregulation of Smad3/Fli-1.

\section{Astragaloside IV regulated Smad3 activity and localization}

To further confirm the inhibitory effect of Astragaloside IV on TGF- $\beta$-Smad pathway, we investigated the activity and localization of Smad3 upon drug treatment. As shown in Fig. 4E, phosphorylated Smad3 (p-SMAD3), which was in activated form in the pathway, was significantly higher in SSc fibroblasts (SScF) compared to normal fibroblasts (NF). Interestingly, treatment with Astragaloside IV not only decreased the level of p-SMAD3, but also completely blocked its relocation into the nucleus where it interacts with other cofactors to activate TGF- $\beta$-dependent gene transcription. 


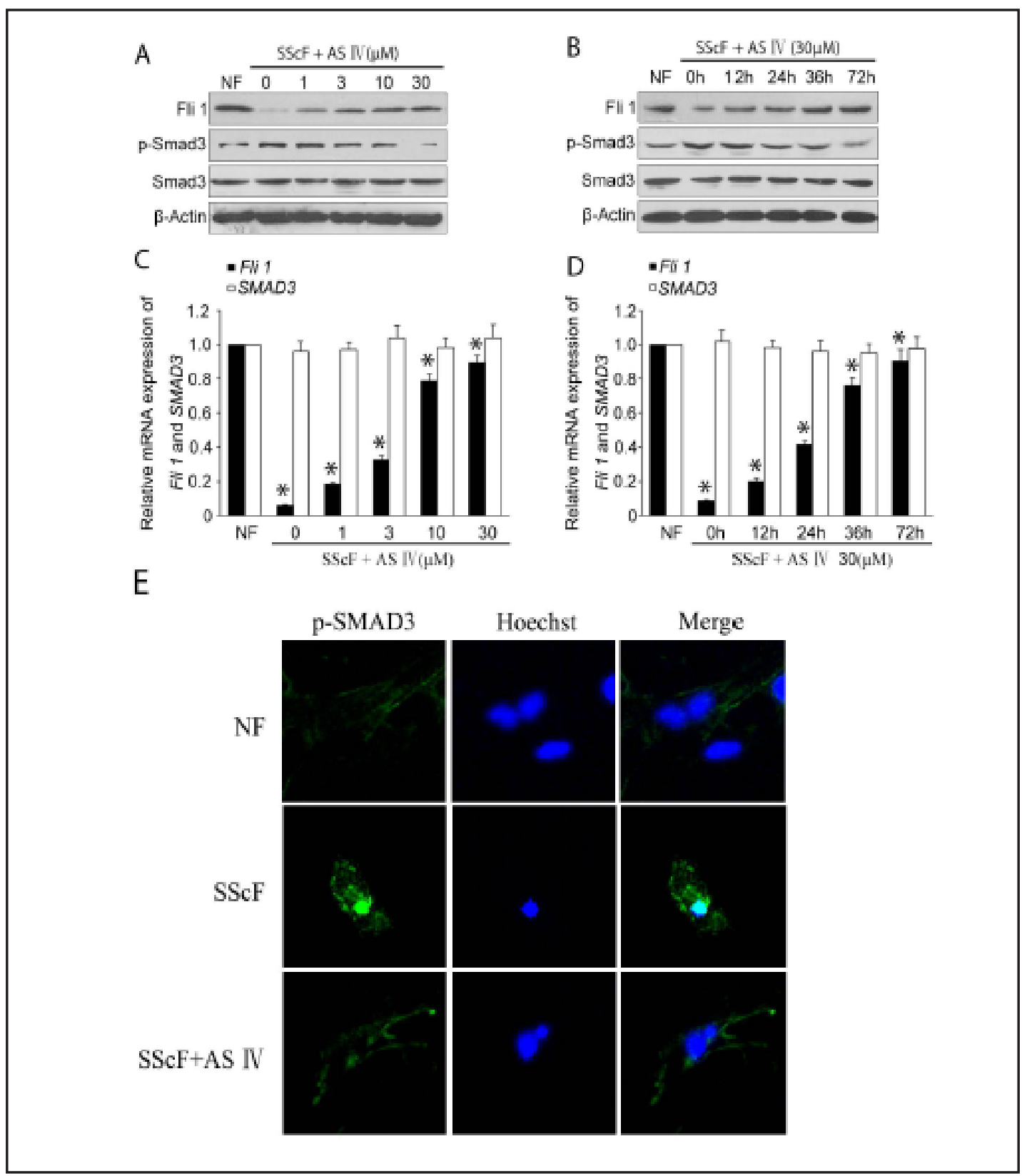

Fig. 4. Astragaloside IV-induced fibrosis reduction is due to deregulation of Smad3/ Fli-1. Normal fibroblasts (NF) and SSc fibroblasts (SScF) were treated with Astragaloside IV (AST) at a series of doses (0, 1, 3, $10,30 \mu \mathrm{M}$ for $72 \mathrm{~h})$ and durations $(0,12,24,36,72 \mathrm{~h}$ at the dose of $30 \mu \mathrm{M})$, followed by western blot and RTqPCR assay. Phosphorylation of Smad3 was markedly increased in SSc fibroblasts than in normal fibroblasts, and was suppressed by Astragaloside IV in dose- (A) and time- (B) dependent manner, while the expression of Fli-1 displayed the opposite trend. No significant change was observed on total Smad3 expression. Similar results were obtained on their mRNA expression level (C, D). RT-qPCR results are expressed as means \pm SD of three independent experiments analyzed with ANOVA. ${ }^{*} p<0.05$ vs SScF + AS IV $(0 \mu \mathrm{M}), \# p<0.05$ vs NF. NF, normal fibroblast; SScF, SSc fibroblast; AS IV, Astragaloside IV. (E) Astragaloside IV regulated Smad3 activity and localization. Normal fibroblast (NF) and SSc fibroblast (SScF) were treated with Astragaloside IV (AST) for $24 \mathrm{~h}$ at a dose of $30 \mu \mathrm{M}$, followed by immunofluorescence staining. Phosphorylated Smad3 (p-SMAD3), was significantly higher in SScF compared to that of NF. Astragaloside IV treatment decreased the level of p-SMAD3 and completely blocked its relocation into the nucleus. NF, normal fibroblast; SScF, SSc fibroblast; AS IV, Astragaloside IV. 


\section{Discussion}

This study is the first, to our knowledge, to report the anti-fibrotic role of Astragaloside IV in systemic sclerosis (SSc) in vitro and in vivo models.

SSc is a complex multi-organ system group of diseases of unknown causative factor [25]. Characteristic features include vasculopathy and excessive collagen accumulation in skin and internal organs [26]. Fibrosis, whereby the deposition of collagen and other components ultimately replaces the normal architecture of blood vessels, internal organs and skin, is considered to be the hallmark of SSc $[27,28]$. Indeed, we isolated SSc fibroblasts from patients and found that expressed levels of collagen and fibronectin, which indicate fibrosis, were significantly higher. Later, in lesional skin samples from SSc model mice, we not only confirmed the increased expression of collagen and fibronectin, but also observed a disordered histology structure. These changes lead to induration, or thickening of the skin, which is the classic clinical symptom [26]. However, the pathogenesis of SSc, despite significant recent developments by dedicated translational scientists, remains largely unknown [29].

Interestingly, Astragaloside IV, one of the most active compounds isolated from a Chinese medical herb [30], could decrease the expression of collagen and fibronectin in a dose- and time-dependent manner. Further, this drug displayed a strong anti-fibrosis effect in vivo, consistent with previous studies $[10,11,15,16]$. Recent studies also suggest that Astragaloside IV has broad application prospects, especially in cardiovascular diseases, digestive diseases, cancer, and other modern high-incidence, high-risk diseases [31]. Nevertheless, the molecular mechanisms of the action of Astragaloside IV are still not clear. Increasing evidence suggests that TGF- $\beta$ plays a key role in the development of SSc [32]. Overexpression of TGF- $\beta$-dependent genes has also been found in biopsy specimens from patients with SSc. Upregulation of the TGF- $\beta$-Smad signaling pathway has been described [33]. TGF- $\beta$ stimulates fibroblast proliferation, differentiation, and cytokine secretion [34]. It promotes the upregulation of collagen synthesis and the accumulation of extracellular matrix (ECM) [35]. TGF- $\beta$ binds to its receptor and activates the signaling leading to phosphorylation of transcription factors Smad2 or Smad3. Phosphorylated Smad2 or Smad3 bind to Smad4, the common Smad, and the resulting complex moves from the cytoplasm into the nucleus [36]. In the nucleus, the Smad complex interacts with various other transcription factors and then transactivates fibrosis-associated genes such as type I collagen and fibronectin [33]. Consistent with these findings, we demonstrated that Astragaloside IV regulated the expression of Smad3/Fli-1, major mediators of the fibrotic response and key molecules for TGF- $\beta$ signaling. Furthermore, Astragaloside IV decreased the level of phosphorylated Smad3 and completely blocked its relocation into the nucleus, where it interacts with other co-factors to activate TGF- $\beta$-dependent gene transcription [37]. Studies suggest that Smad4 interaction with specific transcriptional factors including Sp1, which plays an essential role in the stimulation of collagen transcription in normal fibroblasts, may account for the pro-fibrotic state in SSc [38]. In addition, evidence shows that increased levels of Smad2/3 phosphorylation may be explained by decreased levels of the inhibitory Smad7 in SSc [39]. It would be helpful to illustrate whether Astragaloside IV treatment affects these activating or inhibitory factors in future studies. Intriguingly, Astragaloside IV synergizes with ferulic acid to inhibit renal tubulointerstitial fibrosis in rats with obstructive nephropathy [40]. Thus, another field that requires more exploration may be the combination effect of Astragaloside IV with conventional agents for SSc treatment. One of the limitations of this study is that as well as TGF- $\beta$-Smad signaling, Astragaloside IV may also target other pathways related to SSc, since at least 39 putative targets have been identified by Zhao and his colleagues [41]. Their results suggest that the therapeutic effects of Astragaloside IV are based upon a combination of blocking calcium influx, vasodilation, anti-thrombosis, anti-oxidation, antiinflammation and immune regulation.

In China, treating scleroderma using the prescription for nourishing the kidney and strengthening the essence in traditional Chinese herbal medicine has a long history 
and is effective. The complexity of the composition of Chinese herbal medicine, however, is an obstacle to researches on its therapeutic mechanisms. Astragaloside IV is extracted from Astragalus which is a constuent of the prescription for nourishing the kidney and strengthening the essence. By proving the anti-fibrotic effects of Astragaloside IV on scleroderma, this study elaborated, in a new perspective, the mechanisms of treating scleroderma with the traditional remedy - the prescription for nourishing the kidney and strengthening the essence.

\section{Conclusion}

in this study, we demonstrated the anti-fibrosis effects of Astragaloside IV (AST) in SSc, in vitro and in vivo, which may be due to its regulation of TGF- $\beta$-Smads3 signaling. These data suggest that Astragaloside IV could be a potential drug and novel therapeutic strategy for the treatment of SSc.

\section{Acknowledgments}

This study was supported by National Natural Science Foundation of China (No.81102599), Guangdong Natural Science Foundation (No.10451040701005294) and 2012 Excellent Young Scientist Foundation from Guangzhou University of Chinese Medicine. We are grateful to 91SCI Company for language editing assistance.

\section{Disclosure Statement}

The authors declare that no conflicts of interest exist.

\section{References}

1 Yasuoka H, Yamaguchi Y, Feghali-Bostwick CA: The membrane-associated adaptor protein dok5 is upregulated in systemic sclerosis and associated with igfbp-5-induced fibrosis. PLoS One 2014;9:e87754.

- Lonzetti LS, Joyal F, Raynauld JP, Roussin A, Goulet JR, Rich E, Choquette D, Raymond Y, Senecal JL: Updating the american college of rheumatology preliminary classification criteria for systemic sclerosis: addition of severe nailfold capillaroscopy abnormalities markedly increases the sensitivity for limited scleroderma. Arthritis Rheum 2001;44:735-736.

- 3 Rimar D, Rosner I, Nov Y, Slobodin G, Rozenbaum M, Halasz K, Haj T, Jiries N, Kaly L, Boulman N, Daood $\mathrm{R}$, Vadasz Z: Brief report: lysyl oxidase is a potential biomarker of fibrosis in systemic sclerosis. Arthritis Rheumatol 2014;66:726-730.

4 Ray K: Connective tissue diseases: integrins crucial for the onset of fibrosis in systemic sclerosis--a new therapeutic target? Nat Rev Rheumatol 2013;9:637.

Flanders KC: Smad3 as a mediator of the fibrotic response. Int J Exp Pathol 2004;85:47-64. Christmann RB, Sampaio-Barros P, Stifano G, Borges CL, de Carvalho CR, Kairalla R, Parra ER, Spira A, Simms R, Capellozzi VL, Lafyatis R: Association of interferon- and transforming growth factor betaregulated genes and macrophage activation with systemic sclerosis-related progressive lung fibrosis. Arthritis Rheumatol 2014;66:714-725.

7 Wu M, Schneider DJ, Mayes MD, Assassi S, Arnett FC, Tan FK, Blackburn MR, Agarwal SK: Osteopontin in systemic sclerosis and its role in dermal fibrosis. J Invest Dermatol 2012;132:1605-1614.

8 Lakos G, Takagawa S, Chen SJ, Ferreira AM, Han G, Masuda K, Wang XJ, DiPietro LA, Varga J: Targeted disruption of tgf-beta/smad3 signaling modulates skin fibrosis in a mouse model of scleroderma. Am J Pathol 2004;165:203-217. 


\section{Cellular Physiology Cell Physiol Biochem 2014;34:2105-2116 and Biochemistry

-9 Jia RZ, Jiang L, Qiao LX, Chen PS: [neuroprotective effects of astragulus membranaceus on hypoxia-ischemia brain damage in neonatal rat hippocampus]. Zhongguo Zhong Yao Za Zhi 2003;28:1174-1177.

10 Gui D, Huang J, Guo Y, Chen J, Chen Y, Xiao W, Liu X, Wang N: Astragaloside iv ameliorates renal injury in streptozotocin-induced diabetic rats through inhibiting nf-kappab-mediated inflammatory genes expression. Cytokine 2013;61:970-977.

11 Chen P, Xie Y, Shen E, Li GG, Yu Y, Zhang CB, Yang Y, Zou Y, Ge J, Chen R, Chen H: Astragaloside iv attenuates myocardial fibrosis by inhibiting tgf-beta1 signaling in coxsackievirus b3-induced cardiomyopathy. Eur J Pharmacol 2011;658:168-174.

-12 Du Q, Chen Z, Zhou LF, Zhang Q, Huang M, Yin KS: Inhibitory effects of astragaloside iv on ovalbumininduced chronic experimental asthma. Can J Physiol Pharmacol 2008;86:449-457.

13 Yang Q Lu JT, Zhou AW, Wang B, He GW, Chen MZ: Antinociceptive effect of astragalosides and its mechanism of action. Acta Pharmacol Sin 2001;22:809-812.

14 Liu H, Wei W, Sun WY, Li X: Protective effects of astragaloside iv on porcine-serum-induced hepatic fibrosis in rats and in vitro effects on hepatic stellate cells. J Ethnopharmacol 2009;122:502-508.

-15 Li X, Wang X, Han C, Wang X, Xing G, Zhou L, Li G, Niu Y: Astragaloside iv suppresses collagen production of activated hepatic stellate cells via oxidative stress-mediated p38 mapk pathway. Free Radic Biol Med 2013;60:168-176.

16 Chen X, Peng LH, Shan YH, Li N, Wei W, Yu L, Li QM, Liang WQ, Gao JQ: Astragaloside iv-loaded nanoparticleenriched hydrogel induces wound healing and anti-scar activity through topical delivery. Int J Pharm 2013;447:171-181.

17 Chen X, Peng LH, Li N, Li QM, Li P, Fung KP, Leung PC, Gao JQ: The healing and anti-scar effects of astragaloside iv on the wound repair in vitro and in vivo. J Ethnopharmacol 2012;139:721-727.

18 Masi AT: Preliminary criteria for the classification of systemic sclerosis (scleroderma). Subcommittee for scleroderma criteria of the american rheumatism association diagnostic and therapeutic criteria committee. Arthritis Rheum 1980;23:581-590.

19 Yamamoto T, Nishioka K: Animal model of sclerotic skin. Vi: evaluation of bleomycin-induced skin sclerosis in nude mice. Arch Dermatol Res 2004;295:453-456.

20 Liu S, Li Y, Zhang Z, Xie F, Xu Q, Huang X, Huang J, Li C: Alpha1-adrenergic receptors mediate combined signals initiated by mechanical stretch stress and norepinephrine leading to accelerated mouse vein graft atherosclerosis. J Vasc Surg 2013;57:1645-1656, 1651-1656.

21 Li Y, Sun XX, Elferich J, Shinde U, David LL, Dai MS: Monoubiquitination is critical for ovarian tumor domain-containing ubiquitin aldehyde binding protein 1 (otub1) to suppress ubch5 enzyme and stabilize p53 protein. J Biol Chem 2014;289:5097-5108.

22 Qi Q, Guo Q, Tan G, Mao Y, Tang H, Zhou C, Zeng F: Predictors of the scleroderma phenotype in fibroblasts from systemic sclerosis patients. J Eur Acad Dermatol Venereol 2009;23:160-168.

-23 He WL, Li YH, Yang DJ, Song W, Chen XL, Liu FK, Wang Z, Li W, Chen W, Chen CY, He YL, Zhan WH: Combined evaluation of centromere protein h and ki-67 as prognostic biomarker for patients with gastric carcinoma. Eur J Surg Oncol 2013;39:141-149.

-24 Li Y, Liu S, Zhang Z, Xu Q Xie F, Wang J, Ping S, Li C, Wang Z, Zhang M, Huang J, Chen D, Hu L, Li C: Rage mediates accelerated diabetic vein graft atherosclerosis induced by combined mechanical stress and ages via synergistic erk activation. PLoS One 2012;7:e35016.

25 Tyndall AJ, Bannert B, Vonk M, Airo P, Cozzi F, Carreira PE, Bancel DF, Allanore Y, Muller-Ladner U, Distler O, Iannone F, Pellerito R, Pileckyte M, Miniati I, Ananieva L, Gurman AB, Damjanov N, Mueller A, Valentini G, Riemekasten G, Tikly M, Hummers L, Henriques MJ, Caramaschi P, Scheja A, Rozman B, Ton E, Kumanovics G, Coleiro B, Feierl E, Szucs G, Von Muhlen CA, Riccieri V, Novak S, Chizzolini C, Kotulska A, Denton C, Coelho PC, Kotter I, Simsek I, de la Pena LP, Hachulla E, Seibold JR, Rednic S, Stork J, Morovic-Vergles J, Walker UA: Causes and risk factors for death in systemic sclerosis: a study from the eular scleroderma trials and research (eustar) database. Ann Rheum Dis 2010;69:1809-1815.

26 Abraham DJ, Krieg T, Distler J, Distler O: Overview of pathogenesis of systemic sclerosis. Rheumatology (Oxford) 2009;48:i3-i7.

27 Gabrielli A, Avvedimento EV, Krieg T: Scleroderma. N Engl J Med 2009;360:1989-2003.

28 Valentini G, Cuomo G, Abignano G, Petrillo A, Vettori S, Capasso A, Cozzolino D, Del GG, Santoriello C: Early systemic sclerosis: assessment of clinical and pre-clinical organ involvement in patients with different disease features. Rheumatology (Oxford) 2011;50:317-323. 


\section{Cellular Physiology Cell Physiol Biochem 2014;34:2105-2116 and Biochemistry

29 Saketkoo LA, Magnus JH, Doyle MK: The primary care physician in the early diagnosis of systemic sclerosis: the cornerstone of recognition and hope. Am J Med Sci 2014;347:54-63.

30 Tan S, Wang G, Guo Y, Gui D, Wang N: Preventive effects of a natural anti-inflammatory agent, astragaloside iv, on ischemic acute kidney injury in rats. Evid Based Complement Alternat Med 2013;2013:284025.

31 Ren S, Zhang H, Mu Y, Sun M, Liu P: Pharmacological effects of astragaloside iv: a literature review. J Tradit Chin Med 2013;33:413-416.

- 32 Ihn H: Autocrine tgf-beta signaling in the pathogenesis of systemic sclerosis. J Dermatol Sci 2008;49:103113.

-33 Takagi K, Kawaguchi Y, Kawamoto M, Ota Y, Tochimoto A, Gono T, Katsumata Y, Takagi M, Hara M, Yamanaka $\mathrm{H}$ : Activation of the activin a-alk-smad pathway in systemic sclerosis. J Autoimmun 2011;36:181-188.

34 Varga JA, Trojanowska M: Fibrosis in systemic sclerosis. Rheum Dis Clin North Am 2008;34:115-143.

35 Das D, Holmes A, Murphy GA, Mishra K, Rosenkranz AC, Horowitz JD, Kennedy JA: Tgf-beta1-induced mapk activation promotes collagen synthesis, nodule formation, redox stress and cellular senescence in porcine aortic valve interstitial cells. J Heart Valve Dis 2013;22:621-630.

-36 Miyazawa K, Shinozaki M, Hara T, Furuya T, Miyazono K: Two major smad pathways in tgf-beta superfamily signalling. Genes Cells 2002;7:1191-1204.

37 Hatton N, Frech T, Smith B, Sawitzke A, Scholand MB, Markewitz B: Transforming growth factor signalling: a common pathway in pulmonary arterial hypertension and systemic sclerosis. Int J Clin Pract Suppl 2011:35-43.

-38 Ghosh AK, Quaggin SE, Vaughan DE: Molecular basis of organ fibrosis: potential therapeutic approaches. Exp Biol Med (Maywood) 2013;238:461-481.

-39 Zhou H, Zou S, Lan Y, Fei W, Jiang R, Hu J: Smad7 modulates tgfbeta signaling during cranial suture development to maintain suture patency. J Bone Miner Res 2014;29:716-724.

-40 Meng LQ Tang JW, Wang Y, Zhao JR, Shang MY, Zhang M, Liu SY, Qu L, Cai SQ, Li XM: Astragaloside iv synergizes with ferulic acid to inhibit renal tubulointerstitial fibrosis in rats with obstructive nephropathy. Br J Pharmacol 2011;162:1805-1818.

41 Zhao J, Yang P, Li F, Tao L, Ding H, Rui Y, Cao Z, Zhang W: Therapeutic effects of astragaloside iv on myocardial injuries: multi-target identification and network analysis. PLoS One 2012;7:e44938. 